\title{
Course of Atypical Manifestations of a Case of Charcot-Marie-Tooth Disease over 35 Years of Clinical Observation
}

\author{
Giuseppe Lanza $^{1 *}$, Luisa Vinciguerra ${ }^{1}$, Valentina Puglisi ${ }^{1}$, Daniela Modica ${ }^{1}$, Alfio Catalano ${ }^{1}$, \\ Giuseppe Zelante ${ }^{1}$, Riccardo Ricceri ${ }^{1}$, Lorenzo Lupo ${ }^{2}$, Salvatore Giuffrida ${ }^{1}$, Manuela Pennisi ${ }^{3}$ \\ ${ }^{1}$ Department “G. F. Ingrassia”, Section of Neurosciences, University of Catania, Catania, Italy; ${ }^{2}$ Department “G. F. Ingrassia”, MED \\ 01, University of Catania, Catania, Italy; ${ }^{3}$ Department of Chemistry, University of Catania, Catania, Italy. \\ Email: "giuseppelanza2003@yahoo.it
}

Received April 16 ${ }^{\text {th }}, 2013$; revised May 20 ${ }^{\text {th }}, 2013$; accepted June $5^{\text {th }}, 2013$

Copyright (C) 2013 Giuseppe Lanza et al. This is an open access article distributed under the Creative Commons Attribution License, which permits unrestricted use, distribution, and reproduction in any medium, provided the original work is properly cited.

\begin{abstract}
Introduction: Atypical manifestations of peripheral neuropathy are not rare, challenging the differential diagnosis. In the past, the diagnosis of hereditary neuropathy was mainly based on the clinical and electromyographic (EMG) findings and, occasionally, biopsy. Nowadays, the genetic tests allow us to identify more than 40 different genes/loci associated with Charcot-Marie-Tooth (CMT) disease, although some subtypes are clinically indistinguishable. We have followed a patient with a clinical diagnosis of apparent sporadic and atypical CMT and recently diagnosed genetically as distal hereditary motor neuropathy, type V (dHMN-V). Case Report: Thirty-five years ago, a 16 years old patient complained muscular weakness and wasting at the hands small muscles. Sporadic fasciculations were observed whereas deep tendon reflexes and sensation were normal. EMG examination revealed neurogenic muscular denervation in the distribution of C7, C8 and T1 segments bilaterally. Muscular biopsy of the left Biceps Brachii showed rare atrophic fibers and some cellular atypia. The disease has undergone a clinical and EMG progression and diffusion over the years, involving the lower limbs and leading to a bilateral steppage. A more slight diffuse axonal motor neuropathy was also identified in the proband's son and second cousin. The genetic study found a known missense mutation in BSCL2 gene related to a dHMN-V. Interestingly, there was a remarkable intra-familiar phenotypic variability, especially in the clinical onset and severity. Discussion: Atypical manifestations of hereditary neuropathies often overlap with other conditions. The present case highlights how a comprehensive clinical evaluation and a careful follow-up have led to a correct diagnosis even 35 years later and have allowed to identify other affected family members. The apparent lack of familiarity was probably due to the very soft presentation in the proband's relatives. Although the genetic study was not available at that time, the first clinical diagnosis was not disavowed. The main differential diagnoses and a brief review of similar reported cases are discussed.
\end{abstract}

Keywords: Hereditary Neuropathies; Clinical Overlap; Genetic Analysis; Phenotypic Variability

\section{Introduction}

Charcot-Marie-Tooth (CMT) neuropathy refers to a group of hereditary disorders characterized by a chronic motor and sensory polyneuropathy. The affected individual typically has distal muscle weakness and atrophy often associated with mild to moderate distal sensory loss, depressed tendon reflexes, high-arched feet and weak ankle dorsiflexion. However, atypical clinical manifestations are not rare, often making the differential diagnosis with other neurological disorders challenging. For instance,

"Corresponding author. some Hereditary Motor Neuropathies (HMN) presents with distal weakness [1], thus, suggesting a clinical picture resembling CMT disease, although the HMN does not usually show sensory disturbances.

In the past, classification of CMT spectrum disorders was mainly based on the neurophysiological testing and, occasionally, sural nerve or muscle biopsy. Nowadays, genetic testing is available depending on inheritance patterns and molecular genetics and more than 40 different genes/loci are associated with CMT. However, there are areas of overlap between different types of CMT and some subtypes are clinically indistinguishable and are 
designated solely on molecular findings. Establishing the specific cause of CMT hereditary neuropathy for a given individual encompasses the medical history, physical and neurological examination, nerve conduction and electromyographic (EMG) testing, as well as the use of molecular genetic testing when available.

We report a young patient with a clinical diagnosis of apparent sporadic and atypical CMT, clinically followed by the same Neurologist for approximately 35 years and recently diagnosed genetically as distal HMN, type $\mathrm{V}$ (dHMN-V).

\section{Case Report}

About 35 years ago, a young Neurologist diagnosed a sporadic and atypical case of CMT disease in a 16 years old Italian patient (F. C., proband) working as a bartender. He complained muscular weakness at the upper limbs and progressive muscular wasting almost exclusively at the level of small muscles of both hands.

Herein is described the neurological examination as reported at that time. Severe atrophy of the first and second dorsal interosseous muscles of both hands, more evident on the right side, a moderate atrophy of thenar muscles and a slight atrophy of ipothenar muscles without evident trophic abnormalities in other districts were observed. Weakness, more evident in the distal muscle groups, was tested in thumb opposition and in spreading/closing finger movements. Sporadic fasciculations were also observed and deep tendon reflexes were normal and symmetrical at both upper and lower limbs. Hoffman and Babinski responses were not present. Nystagmus, oculomotor deficits, lower cranial nerves impairment and cerebellar signs were also absent. Eye fundi and visual field were normal. There were no gait and standing position disturbances and all types of sensation were intact. The patient underwent to laboratory and instrumental investigations available at that time. Extensive laboratory tests, including muscle enzymes level, were unremarkable. Cerebrospinal fluid examination was normal and no oligoclonal bands were present. Electroencephalogram (EEG), skull X-ray, cervical and thoracic vertebral X-ray and myelography with iodinated contrast material were unremarkable. EMG examination pointed out severe and bilateral neurogenic muscular denervation in the distribution of $\mathrm{C} 7, \mathrm{C} 8$ and $\mathrm{T} 1$ segments, with evidence of fibrillation potentials. Biopsy of the left Biceps Brachii muscle showed rare atrophic fibers tending to angular shape (Figure 1(A)) and areas of both quantitative and qualitative cellular atypia, such as enzymes depletion and intermyofibrillar reticulum distortion at the center of the fibers (Figure 1(B)). The clinical course over the years was characterized by a slow progression of the atrophic deficit at the upper limbs and, gradually, muscular weakness became apparent at the lower limbs,

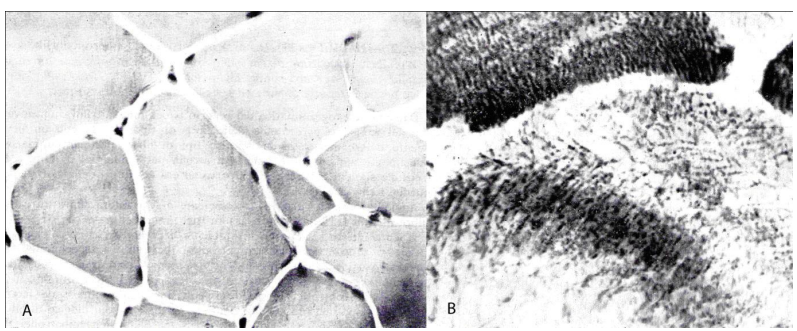

Figure 1. Proband's left Biceps Brachii muscle biopsy (1978). A. Hematoxylin-eosin $\times$ 150: atrophic fibers tending to angular shape; (B) NADH-TR: intermyofibrillar reticulum distortion.

leading to a bilateral steppage.

After 35 years of clinical follow-up, the neurological evaluation showed severe distal muscles atrophy at the four limbs, relevant gait and prehension impairment and slight sensitive impairment. The electroneurographic examination revealed diffuse axonal motor neuropathy, especially at the lower limbs. Thirty-five years after the first observed case, the same Neurologist examined two young patients complaining a recent history of motor clumsiness at both hands, especially when writing. One of them was the proband's son (F. Al., 16 years old) and the other was proband's second cousin (F. An., 14 years old). The EMG results were compatible with an axonal motor neuropathy at the four limbs; median, tibial and peroneal nerves were the most impaired whereas sensory nerves were not involved (Tables 1 and 2). Muscle biopsy was not performed because they underwent to genetic test. The genetic studies led to the definitive diagnosis of dHMN-V. A known missense mutation 263A > $\mathrm{G}$ was identified in BSCL2 gene of the examined patients (proband, F.Al, F.An), resulting in the aminoacid substitution N88S [2,3]. Therefore, clinical, instrumental and genetic tests were performed in other family members. Overall, the genetic study was performed in 13 subjects: the $263 \mathrm{~A}>\mathrm{G}$ missense mutation (AAT > AGT), typically observed in dHMN-V $[1,4]$, was identified in 10 patients. Some of the examined relatives have never consulted a Neurologist because of the very mild clinical presentation with a minimal impairment in the activity of daily living. For instance, the father of the proband's cousin had a subtle atrophy of the hand muscles without any weakness so that he continued to work as a dentist without difficulty.

\section{Discussion}

Distal HMN defines a group of hereditary disorders characterized by distal muscular weakness and atrophy. The subtype dHMN-V affects in particular the upper limbs, especially the small hand muscles. Interestingly, a remarkable intra-familial phenotypic variability in the clinical onset and severity is often observed, thus often 
Table 1. Nerve conduction studies results of the examined patients.

\begin{tabular}{|c|c|c|c|c|c|c|c|c|c|c|c|c|c|c|c|}
\hline \multirow{2}{*}{ Patient } & & \multicolumn{2}{|c|}{$\mathrm{MMN}$} & \multicolumn{2}{|c|}{ UMN } & \multicolumn{2}{|c|}{$\mathrm{PMN}$} & \multicolumn{2}{|c|}{$\mathrm{TMN}$} & \multicolumn{2}{|c|}{ MSN } & \multicolumn{2}{|c|}{ USN } & \multicolumn{2}{|c|}{$\mathrm{SN}$} \\
\hline & & A & $\mathrm{CV}$ & A & $\mathrm{CV}$ & A & $\mathrm{CV}$ & A & $\mathrm{CV}$ & A & $\mathrm{CV}$ & A & $\mathrm{CV}$ & $\mathrm{A}$ & $\mathrm{CV}$ \\
\hline \multirow{2}{*}{ F. C. } & $\mathrm{R}$ & 0.6 & 40 & 11 & 49 & 0.2 & - & 0.1 & 33 & 17 & 39 & 6 & 41 & 11 & 35 \\
\hline & $\mathrm{L}$ & 0.8 & 56 & 11 & 52 & 0.2 & 28 & 0.1 & 33 & 16 & 43 & 7 & 45 & 9 & 35 \\
\hline \multirow{2}{*}{ F. Al. } & $\mathrm{R}$ & 1 & 54 & 17 & 58 & 0.1 & 30 & 1 & 40 & 33 & 49 & 29 & 42 & 14 & 40 \\
\hline & $\mathrm{L}$ & 1 & 57 & 16 & 59 & 0.1 & 29 & 2 & 42 & 49 & 49 & 26 & 41 & 9 & 37 \\
\hline \multirow{2}{*}{ F. An. } & $\mathrm{R}$ & 0.7 & 40 & 15 & 61 & 0.9 & 42 & 10 & 43 & 54 & 43 & 20 & 39 & 18 & 39 \\
\hline & $\mathrm{L}$ & 0.7 & 47 & 9 & 53 & 2 & 50 & 8 & 42 & 45 & 47 & 44 & 43 & 21 & 38 \\
\hline
\end{tabular}

MMN = Median Motor Nerve; MSN = Median Sensory Nerve; UMN = Ulnar Motor Nerve; USN = Ulnar Sensory Nerve; PMN = Peroneal Motor Nerve; TMN $=$ Tibial Motor Nerve; $\mathrm{SN}=$ Sural Nerve. $\mathrm{R}=$ right; $\mathrm{L}=$ left; $\mathrm{A}=$ amplitude: motor $(\mathrm{mV})$; sensory $(\mu \mathrm{V})$; $\mathrm{CV}=$ conduction velocity $(\mathrm{m} / \mathrm{s}) ;-=$ not recordable; bold numbers $=$ abnormal values.

Table 2. Electromyography results of the examined patients.

\begin{tabular}{|c|c|c|c|c|c|c|c|c|c|c|c|c|c|c|c|c|c|}
\hline \multirow[b]{2}{*}{ Patient } & & \multicolumn{2}{|c|}{ BB } & \multicolumn{2}{|c|}{ EDC } & \multicolumn{2}{|l|}{ APB } & \multicolumn{2}{|c|}{ FDI } & \multicolumn{2}{|c|}{$\mathrm{VM}$} & \multicolumn{2}{|l|}{$\mathrm{TA}$} & \multirow{2}{*}{$\frac{\mathrm{MG}}{\mathrm{SA}}$} & \multicolumn{3}{|c|}{ EDB } \\
\hline & & SA & $\mathrm{R}$ & SA & $\mathrm{R}$ & $\mathrm{SA}$ & $\mathrm{R}$ & SA & $\mathrm{R}$ & SA & $\mathrm{R}$ & SA & $\mathrm{R}$ & & $\mathrm{R}$ & SA & $\mathrm{R}$ \\
\hline \multirow{2}{*}{ F. C. } & $\mathrm{R}$ & - & $\mathrm{N}$ & - & $\downarrow$ & - & $\downarrow$ & - & $\downarrow$ & - & $\mathrm{N}$ & - & $\downarrow$ & - & $\downarrow$ & - & $\downarrow$ \\
\hline & $\mathrm{L}$ & - & $\mathrm{N}$ & - & $\downarrow$ & - & $\downarrow$ & - & $\downarrow$ & - & $\mathrm{N}$ & - & $\downarrow$ & - & $\downarrow$ & - & $\downarrow$ \\
\hline \multirow{2}{*}{ F. Al. } & $\mathrm{R}$ & - & $\mathrm{N}$ & - & $\downarrow$ & $+\mathrm{F}$ & $\downarrow$ & $+\mathrm{F}$ & $\downarrow$ & - & $\mathrm{N}$ & $+\mathrm{F}$ & $\downarrow$ & $\mathrm{F}$ & $\downarrow$ & - & $\downarrow$ \\
\hline & $\mathrm{L}$ & - & $\mathrm{N}$ & - & $\downarrow$ & $+\mathrm{F}$ & $\downarrow$ & $+\mathrm{F}$ & $\downarrow$ & - & $\mathrm{N}$ & $+\mathrm{F}$ & $\downarrow$ & F & $\downarrow$ & - & $\downarrow$ \\
\hline \multirow{2}{*}{ F. An. } & $\mathrm{R}$ & - & $\mathrm{N}$ & - & $\downarrow$ & + & $\downarrow$ & - & $\downarrow$ & - & $\mathrm{N}$ & + & $\downarrow$ & - & $\mathrm{N}$ & + & $\downarrow$ \\
\hline & $\mathrm{L}$ & - & $\mathrm{N}$ & - & $\downarrow$ & + & $\downarrow$ & - & $\downarrow$ & - & $\mathrm{N}$ & + & $\downarrow$ & - & $\mathrm{N}$ & + & $\downarrow$ \\
\hline
\end{tabular}

$\mathrm{BB}=$ biceps brachii $\mathrm{EDC}=$ extensor digitorum communis; $\mathrm{APB}=$ abductor pollicis brevis; $\mathrm{FDI}=$ first dorsal interosseous; $\mathrm{VM}=\mathrm{vastus}$ medialis; $\mathrm{TA}=$ tibialis anterior; $\mathrm{MG}=$ medial gastrocnemius; $\mathrm{EDB}=$ extensor digitorum brevis; $\mathrm{SA}=$ spontaneous activity; $\mathrm{R}=$ recruitment; $-=$ absent; $\mathrm{N}=$ normal; + = fibrillations; $\mathrm{F}=$ fasciculations; $\downarrow=$ reduced. $\mathrm{R}=$ right; $\mathrm{L}=$ left;

challenging the differential diagnosis.

In this context, the main differential diagnosis to be taken into account is the Progressive Muscular Atrophy, a rare subtype of Amyotrophic Lateral Sclerosis formerly known as "Aran-Duchenne disease" at that time [4]. The clinical presentation, the lack of familiarity and the EMG data seemed to support this hypothesis. However, based on the young age of onset, the non-progressive clinical course as well as the instrumental and neuropathological findings, a diagnosis of lower motor neuron disease was unlikely. At first, inheritance seemed to be excluded since similar clinical presentation was not referred neither in his parents or in other family members. The early upper limbs involvement, and especially the lack of sensory impairment, suggested the diagnosis of sporadic and atypical CMT disease.

Distal HMN-V also shares clinical and genetic features with another hereditary neuropathy, the CMT2D, and with the hereditary spastic paraplegia 17 also known as Silver syndrome. Clinically, CMT2D is characterized by an early onset [6] and a predominant upper limbs involvement in terms of muscular weakness and cramps, progressive wasting of small hand muscles and sensory disturbances, the latter being typically absent in the dHMN-V. Silver syndrome [3], a rare autosomal dominant neurodegenerative disorder, has variable onset and, although presenting with amyotrophy of the small hand muscles, is mainly characterized by spasticity and other pyramidal signs at the lower limbs and, subsequently, foot deformities and slight sensory complaints can also occur [2]

Literature reports some families with a phenotypic and genetic diagnosis of dHMN-V. In 2010 RakocevicStoianovic et al. [7] described the first three Serbian family members with a BSCL2 mutation showing variable expression within the family: a 55-year-old woman with distal muscle weakness and atrophy in her legs and hypereflexia (except for ankle reflexes); her 25 year-old son with lower limbs stiffness and hyporeflexia; her 55 year-old cousin with a more prominent involvement of leg muscles and mild asymmetrical weakness of hand muscles. Sensory nerve conduction velocities were normal whereas compound muscle action potential amplitudes were markedly reduced in all patients; concentric 
needle EMG showed chronic denervation in distal muscles. In 2006, van de Warrenburg et al. [8] reported the first two Dutch families with BSCL2 mutations and underscored the phenotypic spectrum of BSCL2 gene mutations with an overlap between Silver syndrome, dHMN$\mathrm{V}$ and dHMN-II, highlighting the variable phenotypic patterns between and within the families. Despite a rather early onset in the second or first decade, disease progression was usually slow and mildly impacts the daily living functions. The heterogeneous clinical presentations included: foot muscle weakness and atrophy, with or without gradually evolving pyramidal tract signs or hand muscle involvement; predominant involvement of hand muscles, with minimal or no lower limb involvement and the early or late presence of Babinski's sign; predominant spastic paraparesis with minimal distal lower limb involvement. Therefore, although the phenol-type may eventually become compatible with Silver syndrome variant (with predominant foot rather than hand muscle involvement), the dHMN-II or dHMN-V resembling features are often encountered in early stages and pyramidal tract signs might even remain absent.

In a report of 90 patients with the N88S mutation [9], the following distribution of clinical subtypes was observed: $4.4 \%$ (still) unaffected; $20 \%$ subclinically/very mildly affected; $31.1 \%$ dHMN-type $\mathrm{V} ; 14.5 \%$ classical Silver syndrome; $20 \%$ CMT like phenotype (either spinal form or hereditary motor sensory neuropathies-II depending on the absence or presence of sensory abnormalities, respectively); $10 \%$ a pure spastic paraparesis.

More recently, a Chinese family over four generations with seipinopathy was studied [10]. Six family members were dHMN-II with predominant weakness of lower extremities, whereas one was accompanied by pyramidal signs. Other three women were dHMN-V with predominant hands atrophy.

Taken together, these findings interestingly highlight the view that the phenotype of motor neuropathies associated with BSCL2 mutations is broader than Silver syndrome and dHMN-V [1]. In this context, Irobi et al. [1] reported two families, one with members affected by marked spasticity in the lower limbs and very striking distal amyotrophy that always started in the legs and the other with distal amyotrophy sometimes starting and predominating in the legs, but no pyramidal tract signs. Further evidence for genetic heterogeneity of dHMN-V, CMT2 with predominant hand involvement and Silver syndrome came from Rohkamm et al. [11], confirming that most likely only two mutations (N88S, S90L) in exon 3 of BSCL2 may lead to dHMN-V or Silver syndrome phenotypes whereas mutations in other genes (such as GARS, HSPB1 and HSPB8) are not a common cause of dHMN-V, Silver syndrome and CMT2D.

\section{Conclusion}

Some atypical manifestations of CMT disease, such as the apparent lack of familiarity, the predominant upper limbs involvement at the onset, together with no tendon reflexes or sensation abnormalities, intrigued a young Neurologist 35 years ago. Although the genetic study was not available at that time, a comprehensive diagnostic work-up and a continuous clinical observation, in addition to modern advanced diagnostic procedures such as genetic analysis, allowed us to delineate a definitive diagnosis and to identify other family members affected by a rare hereditary neuropathy. The apparent lack of familiarity that led to the diagnosis of atypical and sporadic CMT in the past was probably due to the very soft presentation and course of the disease in the proband's relatives, highlighting the impressive intra-familiar variability that might characterize these conditions. In conclusion, 35 years later, the same Neurologist has not disavowed his first diagnosis.

\section{REFERENCES}

[1] J. Irobi, P. De Jonghe and V. Timmerman, "Molecular Genetics of Distal Hereditary Motor Neuropathies," $\mathrm{Hu}$ man Molecular Genetics, Vol. 13, No. 2, 2004, pp. R195R202.

[2] C. Windpassinger, M. Auer-Grumbach, J. Irobi, H. Patel, E. Petek, G. Horl, et al., "Heterozygous Missense Mutations in BSCL2 Are Associated with Distal Hereditary Motor Neuropathy and Silver Syndrome," Nature Genetics, Vol. 36, No. 3, 2004, pp. 271-276. doi: $10.1038 / \operatorname{lng} 1313$

[3] D. Ito and N. Suzuki, "Seipinopathy: A Novel Endoplasmic Reticulum Stress-Associated Disease," Brain, Vol. 132, 2009, pp. 8-15.

[4] M. Bonduelle, "Aran-Duchenne? Duchenne-Aran? The Quarrel around Progressive Muscular Atrophy," Revue Neurologique Société de Neurologie de Paris, Vol. 146, No. 2, 1990, pp. 97-106.

[5] J. Magrè, M. Delépine, E. Khallout, T. Gedde-Dahl, L. Van Maldergem, E. Sobel, et al., "Identification of the Gene Altered in Berardinelli-Seip Congenital Lipodystrophy on Chromosome 11q13," Nature Genetics, Vol. 28, No. 4, 2001, pp. 365-370. doi:10.1038/ng585

[6] K. Sivakumar, T. Kyriakides, I. Puls, G. A. Nicholson, B. Funalot, A. Antonellis, et al., "Phenotypic Spectrum of Disorders Associated with Glycyl-tRNA Synthetase Mutations," Brain, Vol. 128, No. 10, 2005, pp. 2304-2314. doi:10.1093/brain/awh590

[7] V. Rakocević-Stojanović, V. Milić-Rasić, S. Perić, J. Baets, V. Timmerman, I. Dierick, et al., "N88S Mutation in the BSCL2 Gene in a Serbian Family with Distal Hereditary Motor Neuropathy Type V or Silver Syndrome," Journal of the Neurological Sciences, Vol. 296, No. 1-2, 2010, pp. 107-109. doi:10.1016/j.jns.2010.06.015

[8] B. P. van de Warrenburg, H. Scheffer, J. J. van Eijk, M. H. 
Versteeg, H. Kremer, M. J. Zwarts, et al., "BSCL2 Mutations in Two Dutch Families with Overlapping Silver Syndrome-Distal Hereditary Motor Neuropathy," Neuromuscular Disorders, Vol. 16, No. 2, 2006, pp. 122-125. doi:10.1016/j.nmd.2005.11.003

[9] M. Auer-Grumbach, B. Schlotter-Weigel, H. Lochmüller, G. Strobl-Wildemann, P. Auer-Grumbach, R. Fischer, et al., "Phenotypes of the N88S Berardinelli-Seip Congenital Lipodystrophy 2 Mutation," Annals of Neurology, Vol. 57, No. 3, 2005, pp. 415-424. doi:10.1002/ana.20410

[10] B. Chen, R. Zheng, X. Luan, W. Zhang, Z. Wang and Y.
Yuan, "Clinical and Pathological Study of Distal Motor Neuropathy with N88S Mutation in BSCL2," Neuropathology, Vol. 29, No. 5, 2009, pp. 543-547. doi:10.1111/j.1440-1789.2009.01011.x

[11] B. Rohkamm, M. M. Reilly, H. Lochmüller, B. SchlotterWeigel, N. Barisic, L. Schöls L, et al., "Further Evidence for Genetic Heterogeneity of Distal HMN Type V, CMT2 with Predominant Hand Involvement and Silver Syndrome," Neurological Sciences, Vol. 263, No. 1-2, 2007, pp. 100-106. doi:10.1016/j.jns.2007.06.047 